\title{
Theory of Planned Behavior in Cyberbullying: A Literature Review
}

\author{
Siriporn Santre \\ College of Public Health Sciences, Chulalongkorn University, Bangkok, 10330 Thailand
}

\begin{abstract}
The rising usage of digital devices and the internet has been linked to an increase in cyberbullying. For cyberbullying has been related to a variety of negative outcomes. Using the theory of planned behavior (TPB), this study aimed to identify determinant elements that influence people's intentions to cyberbully others. To review applications of TPB in the domain of cyberbullying and to verify the efficiency of theory to explain and predict cyberbullying behavior. Furthermore, an application of TPB in cyberbullying prevention program was presented.
\end{abstract}

Keywords: Theory of planned behavior, Cyberbullying, Attitude, Subjective norms

\section{INTRODUCTION}

Online communication has evolved into a general type of communication as a result of the obvious increased utilization of the internet and technology. ${ }^{(1)}$ The use of social media has been considered to be one of the most popular activities. The rising usage of digital devices and the internet has been connected to an increase in cyberbullying. ${ }^{(2)}$ Victims of cyberbullying may experience health problems such as weight loss or gain, substance abuse, stomach pain, unhealthy eating, and restlessness. According to several studies, adolescents who have been cyberbullied exhibit greater signs of violent behavior. Furthermore, being a perpetrator has been linked to poor psychological well-being and external issues. $^{(3,4)}$ Despite the rise of cyberbullying, research into the theoretical background of such online behaviors has been limited. However, by employing the theory of planned behavior, the program could more precisely pave the path for a reduction in the number of incidents of cyberbullying behavior.

\section{Theory of planned behavior}

The theory of planned behavior (TPB) developed from the theory of reasoned action. ${ }^{(5)}$ Human behavior is explained by TPB as the result of rational, elaborative thought. ${ }^{(6)}$ Attitudes, subjective norms (SN), and perceived behavioral control (PBC) linked with the behavior are used to predict behavior in TPB. ${ }^{(7)}$ This theory has been mainly used to the analysis of various behaviors and has been used to predict behavior, including health-related activities. According to this theory, a person's belief in a certain behavior, along with subjective norm and perceived behavioral control, were the components that determined whether they intended to perform the behavior or not. The individual's intention to conduct a specific behavior is a crucial aspect in TPB. ${ }^{(8)}$ The intention to conduct the behavior is the immediate antecedent of behavior in the TPB; the greater the intention, the more probably the behavior will follow. The degree to which people have actual control over their behavior is determined by their capacity to overcome such obstacles, as well as the presence of facilitating variables such as prior experience and support from others. ${ }^{(9)}$

The TPB emphasizes on how the impacts on a person influence that person's decision to engage in a particular action. For 
intentions are assumed to acquire the motivation that affect behavior or decision to exert effort to perform behavior. PBC is an individual's anticipation of a process of behavior that is within their control, and it is viewed as moderating the effect on intention to perform. When PBC is strong and represents actual control, intentions have a greater impact. Some behaviors may be influenced by non-motivational variables such as the resource availability and opportunities such as expertise, money, time, and other people's cooperation. These variables show a person's actual control over their behavior. ${ }^{(10)}$

The elements for behavioral achievement include intention (motivation) and behavioral control (ability). Intention is described as evidence of how hard people are willing to try or how much planning effort, they are willing to exert in order to perform the behavior. Perceived behavioral control and intention compliance are used effectively to predict behavioral achievement. Keeping intention constant, the effort expended to bring behavior to successful conclusion is likely to improve perceived behavioral control. A behavior that is considered to be difficult to accomplish has a low degree of perceived behavioral control, whereas one that is perceived to be simple to perform has a high level of perceived behavioral control. A person with high perceived behavioral control over a specific behavior is more likely to create the intention to conduct that behavior than a person with low perceived behavioral control..$^{(8,11)}$

The intentions can be determined by three variables. The first variable is attitudes, which are the individual's overall behavior evaluations. The second type of norm is subjective norms, which comprise an individual's beliefs about whether significant others think they should engage in the behavior. The final component is $\mathrm{PBC}$, which is the individual's perception of how much control they have over the behavior's performance. ${ }^{(9)}$

\section{Attitude}

Attitude is determined by a person's salient behavioral beliefs, which represent the perceived outcomes of the behavior. The concept of attitude refers to the degree to which a person has a positive or negative outcome toward the behavior. Individuals' attitudes are determined by their behavioral beliefs. For the positive or negative outcomes, beliefs that may affect students' attitudes about cyberbullying. For example, one of the main benefits of cyberbullying may be that it raises a student's status in his or her peer group. Furthermore, it may provide a way to eliminate unpleasant feelings (such as revenge, hatred, and envy) or to generate favorable feelings (such as feeling funny and more powerful than other). Rejection by peers and punishment by others may occur as negative outcomes, such as feelings of guilt or the perception that the victim is harmed. ${ }^{(10)}$

\section{Subjective norm}

Subjective norm refers to the social pressure that individuals feel from significant others when they engage in a specific action. Parents, family members, peers, teachers, and reference groups are examples of significant others. Subjective norms are perceived societal pressures to perform or not perform the behavior. Subjective norms are normative beliefs functions that commit the perceptions of specific salient others' perspectives on whether or not a person should or should not conduct a behavior. These beliefs are expectations about whether or not others from different reference groups believe the person should perform the specified behavior. This is characterized in the theory as the subjective likelihood that specified outstanding groups or individuals (referents) believe the person should or should not engage in the behavior. Normative beliefs typically analyze personal beliefs about the acceptability of behaviors; nevertheless, factors from classmates, families, and others may play an essential role for people. ${ }^{(12)}$ 


\section{Perceived behavioral control}

Perceived behavioral control refers to the perceived difficulty or simplicity of performing out a behavior and can be defined as the mechanism through which people perceive control when they intend to engage in a specific action. Internal and external control factors are included in the control factors. The internal control factors (feelings, personal deficiencies, information, powers, abilities) and external control factors (opportunities, others cooperation, barriers). The more inputs or resources and opportunities people believe they have, as well as the fewer constraints they expect, the greater their control over perceptual behavior. The perceived factors are more likely to obstruct or assist the act of the behavior. People who believe they have opportunities and resources to perform the behavior are more likely to believe they have a high level of behavioral control. PBC are influenced by thoughts about access to the necessary opportunities to conduct the behavior achievement, which are defined by the perceived power in each factor. These control beliefs describe how likely a person believes it is that specific factors will make it easier or more difficult to perform behavior. Each control factor is determined by its perceived ability to support or restrain behavior engagement. ${ }^{(8)}$

\section{Theory of Planned Behavior in Cyberbullying}

Before applying this theory to the context of the adolescent as a perpetrator role in cyberbullying, there are two important considerations. First, social elements such as peer influence and bystander involvement are vital in bullying and must be considered when determining cyberbullying. The subjective norm concept in the TPB incorporates this social component. The second thing is to investigate how the three variables of TPB characteristics influence adolescents' behavioral intention to cyberbully. ${ }^{(13)}$

According to the TPB, people's attitudes toward behavior emerge from their behavioral beliefs. Positive outcomes are associated with positive attitudes toward behaviors, and poor outcomes are associated with negative attitudes toward behaviors. People who feel that aggressive action is acceptable in a bullying situation are more likely to behave forcefully. A positive association exists between people scores on a measure of justification for violence, with high scores suggesting persons' tendency to conceive of aggression as acceptable, and their involvement as a perpetrator in cyberbullying. The majority of perpetrators thought that their cyberbullying behaviors were amusing, whereas victims did not find them humorous and perceived them as harmful. ${ }^{(10)}$

The relation in the predictive value of subjective norm on behavioral intention is that the more people believe that their peers think they should act the behavior, the more motivated people are to comply with peer pressure. On the other side, the more people believe that their peers would react negatively, the less motivated they will be to undertake the behavior. In the context of cyberbullying, it has been established that adolescents who have peers who have positive attitudes regarding cyberbullying are more likely to act as perpetrators. Furthermore, the school context is a major predictor of students' engagement as bullies, especially in schools with high rates of violence and teachers who ignore the bullying. It is possible to conclude that cyberbullying among students is significantly connected with normative beliefs determined by peers who accept cyberbullying. ${ }^{(14)}$

Students' perceptions of cyberbullying, students who would not act as conventional bullies become cyberbullies because they believe they are hidden or anonymous, which reduces their fear of being found and publicly penalized. In this circumstance, the capacity to connect anonymously on the internet leads to a decrease in self-awareness in people and causes them to react aggressively to other persons through cyberspace. ${ }^{(15)}$ 
Furthermore, cyberbullying typically occurs without receiving a visual response from the victim, implying that cyberbullies have no witnesses to their actions. When compared to conventional bullying, the $24 / 7$ accessibility to social networks is an extra feature that supports cyberbullying. Cyberbullies no longer have a restricted amount of place and time to reach their victims. $^{(16)}$

The content they are exposed to on social networking sites (SNSs) will determine certain norms and individuals' confidence in their ability to intervene in cyberbullying. The majority of SNSs include built-in algorithms that generate personalized content based on user preferences. ${ }^{(17)}$ Examining how adolescents view the consequences and outcomes, such as raising awareness, is important for understanding attitudes toward cyberbullying behavior on the internet. For subjective norms, it is designed to investigate the normative impact of friends on students' attitudes regarding cyberbullying, such as a program to improve classroom atmosphere or social responsibility. For perceived behavioral control, it was investigated how students feel about controlling their social networks, such as, helping methods, gaining experience, or self-protection. In terms of behavioral intention, it was investigated how hard students are willing to try to stop cyberbullying behavior. The intention, which is defined by student attitudes. ${ }^{(18)}$

According to the findings in Malaysia, subjective norms are the most important predictor for intention toward cyberbullying behavior, followed by attitudes. PBC is the weakest aspect in cyberbullying, although it does contribute to facilitate motivation to perform behavior. As a result of the technological control, it is probable that students perceived the behavior as simple. ${ }^{(19)}$ The prevention program for this outcome should focus on changing students' positive attitudes toward cyberbullying into negative attitudes, and such interventions are critical for improving the need to understand the consequences of cyberbullying behavior to the victims. ${ }^{(13)}$

The anonymity provided by the internet, combined with the lower chance of being detected, may be a motivating factor in cyberbullying. Additionally, no personal or face-to-face communication is necessary, which may lead people to lose control and act in inappropriate behaviors. ${ }^{(13)}$ This theory is suitable for the period of an individual's development when peer power is most important to them as a strong subjective norm to their motivation to perform. TPB would thus examine cyberbullying attitudes, whereas $\mathrm{SN}$ and PBC would influence and impact cyberbullying intentions and behaviors. ${ }^{(20)}$

\section{Theory of Planned Behavior in cyberbullying prevention program}

The theory of planned behavior was used to demonstrate cyberbullying perpetration in order to perform intervention and prevention of cyberbullying behaviors. TPB may provide information about cyberbullying study and policymakers. In Germany, for example, five school students participated in an intervention program to prevent cyberbullying. Using the theory of planned behavior, the intervention was aimed to reduce cyberbullying behavior. The training includes psychosocial support, legal rights, internet security, and interpersonal skills. The programs are intended to raise awareness and attitudes regarding the legal and social consequences of cyberbullying. The classroom environment has been improved, and students' behavioral control has been increased. When faced with cyberbullying, the provision of online protective and supportive solutions. All of the lessons will lead in a desire to reduce cyberbullying behavior. A 10-week extended version with 90-minute sessions per week was available, as was a one-day abbreviated version with four sessions. The intervention demonstrated that intervention courses could lower cyberbullying behavior when compared to control groups. ${ }^{(18)}$ 
The purpose of this study is to assess the effectiveness of a Safe Surfing anticyberbullying intervention program based on the theory of planned behavior in decreasing bullying offline and online and increasing student-perceived popularity and self-esteem. It was hypothesized that from baseline to post-test online and offline bullying rates will decrease; negative perception of popularity will decrease; selfesteem of student will increase, and in classrooms where there has been a greater reduce of bullying and cyberbullying following the intervention there will be a greater improvement in students' negative perception of popularity and self-esteem. Data were obtained from 1,550 students (53\% in males) from 3rd to 11th grades with 69 classes in 19 primary (68\%), middle and high (32\%) public schools in Israel. The students were completed online questionnaires baseline and post-test. The findings revealed a significant decrease in cyberbullying and bullying at post-test. Also, a significant increasing in perceived popularity and self-esteem was obtained among primary school students. The decrease in cyberbullying and bullying was significantly associated with an increasing in perceived popularity and self-esteem. The study provides support for the positive role that school-based interventions against cyberbullying can have and illustrates that schools can make a difference in the way their students consume social networks. The results also contribute to the debate about the co-occurrence between cyberbullying and conventional bullying. ${ }^{(21)}$

\section{CONCLUSION AND RECOMMENDATIONS}

The purpose of this study was to determine the impact of theory of planned behavior factors such as attitude, subjective norm, and perceived behavioral control on the intention to cyberbully. Evidence on the TPB's applicability in the context of cyberbullying can be found in this literature. TPB creates a good theoretical framework for predicting cyberbullying perpetration.
The most important predictor of cyberbullying perpetration is attitude, subjective norm, or perceived behavioral control. To prevent cyberbullying behaviors, preventive interventions should include activities to boost self-esteem, creates a good attitude, and subjective norm. TPB and the usage of social media should be considered when planning and creating appropriate interventions to minimize and eliminate cyberbullying among all parties in the educational and health sectors, both public and private, in order to promote and advocate the anti-cyberbullying program.

\section{Acknowledgement: None}

\section{Conflict of Interest: None}

\section{Source of Funding: None}

\section{REFERENCES}

1. Ovigli, Junior. Information and Communication Technologies (ICT) in educational research in science museums in Brazil. The International Journal of Education and Development using Information and Communication Technology. 2020;16:272-86.

2. Eleuteri, Saladino, Verrastro. Identity, relationships, sexuality, and risky behaviors of adolescents in the context of social media. Sexual and Relationship Therapy. 2017;32(3-4):354-65.

3. Ybarra, Diener-West, Leaf. Examining the overlap in internet harassment and school bullying: implications for school intervention. J Adolesc Health. 2007;41(6 Suppl 1):S42-50.

4. Mishna, Khoury-Kassabri, Gadalla, Daciuk. Risk factors or involvement in cyber bullying: Victims, bullies and bully-victims. Children and Youth Services Review CHILD YOUTH SERV REV. 2012;34.

5. Ajzen. From Intentions to Actions: A Theory of Planned Behavior. In: Kuhl J, Beckmann J, editors. Action Control: From Cognition to Behavior. Berlin, Heidelberg: Springer Berlin Heidelberg; 1985. p. 11-39.

6. Manfredo. Who Cares About Wildlife?: Social Science Concepts for Exploring Human-Wildlife Relationships and 
Conservation Issues: New York: Springer Press; 2008.

7. Fishbein, Ajzen. Predicting and Changing Behavior: The Reasoned Action Approach. 1st Edition ed: Psychology Press.; 2009.

8. Ajzen. The theory of planned behavior. Organizational Behavior and Human Decision Processes. 1991;50(2):179-211.

9. Ajzen. The theory of planned behavior: Frequently asked questions. Human Behavior and Emerging Technologies. 2020;2(4):314-24.

10. Pabian, Vandebosch. Using the theory of planned behaviour to understand cyberbullying: The importance of beliefs for developing interventions. European Journal of Developmental Psychology. 2014;11: 463-77.

11. Ajzen, Gilbert Cote. Attitudes and the prediction of behavior. In: Crano, Prislin, editors. Attitudes and persuasion: New York: Psychology Press; 2008. p. 289-311.

12. Raskauskas, Stoltz. Involvement in traditional and electronic bullying among adolescents. Developmental psychology. 2007;43:564-75.

13. Heirman, Walrave. Predicting adolescent perpetration in cyberbullying: an application of the theory of planned behavior. Psicothema. 2012;24(4):614-20.

14. Cantone, Piras, Vellante, Preti, Daníelsdóttir, D'Aloja. Interventions on bullying and cyberbullying in schools: a systematic review. Clin Pract Epidemiol Ment Health. 2015;11(Suppl 1 M4):58-76.

15. Kowalski, Limber, Agatston. Cyber Bullying: Bullying in the Digital Age. American Journal of Psychiatry - AMER J PSYCHIAT. 2012;165.

16. Kowalski, Limber. Electronic Bullying Among Middle School Students. The
Journal of adolescent health: official publication of the Society for Adolescent Medicine. 2008;41:S22-30.

17. Leung, Wong, Farver. Cyberbullying in Hong Kong Chinese students: Life satisfaction, and the moderating role of friendship qualities on cyberbullying victimization and perpetration. Personality and Individual Differences. 2018;133:7-12.

18. Wölfer, Schultze-Krumbholz, Zagorscak, Jäkel, Göbel, Scheithauer. Prevention 2.0: targeting cyberbullying @ school. Prev Sci. 2014;15(6):879-87.

19. Shawal, Rashid, Mohamed, Azman. Predicting the intention to cyberbully and cyberbullying behaviour among the undergraduate students at the International Islamic University Malaysia. International Journal of Education, Psychology and Counseling. 2017;2:257-70.

20. Auemaneekul, Powwattana, Kiatsiri, Thananowan. Investigating the mechanisms of theory of planned behavior on Cyberbullying among Thai adolescents. Journal of Health Research. 2019; ahead-ofprint.

21. Aizenkot, Kashy-Rosenbaum. The effectiveness of safe surfing, an anticyberbullying intervention program in reducing online and offline bullying and improving perceived popularity and selfesteem. Cyberpsychology: Journal of Psychosocial Research on Cyberspace. 2020;14.

How to cite this article: Santre S. Theory of planned behavior in cyberbullying: a literature review. International Journal of Research and Review. 2021; 8(11): 234-239. DOI: https://doi. org/10.52403/ijrr.20211131 